\title{
Gaugino condensation and geometry of the perfect square
}

\author{
Renata Kallosh* \\ Stanford Institute for Theoretical Physics and Department of Physics, \\ Stanford University, Stanford, California 94305, USA
}

(Received 30 January 2019; published 13 March 2019)

\begin{abstract}
Gaugino condensation plays a crucial role in the formation of de Sitter vacua. However, the theory of this effect is still incomplete. In four dimensions, the perfect square nature of gaugino couplings follows from the square of auxiliaries in the supergravity action. We explain here why the supersymmetric non-Abelian Dp-brane action, which is the basis for the theory of ten-dimensional gaugino condensation, must have a fourgaugino coupling. This term in the Einstein-Yang-Mills ten-dimensional supergravity is a part of the perfect square, mixing a 3-form with a gaugino bilinear. The perfect square term follows from the superspace geometry, being a square of the superspace torsion. The supercovariant equation of motion for a gaugino on the non-Abelian Dp-brane also involves a supertorsion in agreement with the perfect square term in the action.
\end{abstract}

DOI: 10.1103/PhysRevD.99.066003

\section{INTRODUCTION}

The issue of supersymmetry in Dp-brane actions is well understood for a single Dp-brane. The corresponding action constructed in Refs. [1-3] has a local fermionic $\kappa$ symmetry. When this local $\kappa$ symmetry is gauge fixed as in Refs. [4,5], one recovers the Abelian supersymmetric Dpbrane action, which breaks the maximal supersymmetry of type-II supergravity, half of it being realized linearly.

In [6-10] the coupling of the background 3-form field to the bilinear of the fermions was established for Abelian supersymmetric Dp-branes. The four-fermion nonderivative coupling is absent in the Abelian Dp-brane action, as one can see by a direct inspection of the gauge-fixed $\kappa$-symmetric brane action [1-5].

The concept of gaugino condensation in ten dimensions is based on properties of $\mathrm{N}$ coincident D7-branes. The problem of finding the supersymmetric non-Abelian action for the $\mathrm{N}$ coincident Dp-branes is not fully solved yet. In particular, the four-fermion nonderivative coupling was not investigated in Refs. [6-10] in this context.

This issue attracted some attention after the recent ten-dimensional (10D) investigation of the Kachru, Kallosh, Linde, Trivedi (KKLT) mechanism [34] in Ref. [11] (see also Ref. [12]). This investigation was based on a number of assumptions debated in Refs. [13-16], and the final conclusions of Refs. [11,12] turned out to be

\footnotetext{
*kallosh@stanford.edu
}

Published by the American Physical Society under the terms of the Creative Commons Attribution 4.0 International license. Further distribution of this work must maintain attribution to the author(s) and the published article's title, journal citation, and DOI. Funded by SCOAP. inconsistent with the results of the four-dimensional (4D) KKLT analysis [15-17].

One of the assumptions made in Refs. $[11,12]$ was the absence of the four-fermion nonderivative coupling on D7-branes. This assumption was recently challenged in Ref. [18]. The proposal of how to incorporate such terms, which was made in Ref. [18], was motivated by the heterotic string theory and Horava-Witten model [19-21], which both have the corresponding four-gaugino couplings associated with the perfect square structure of Einstein-Yang-Mills supergravity in ten dimensions.

Here, we will explain why the non-Abelian generalization of Dp-brane action must have a four-fermion nonderivative coupling. We will also show that the supersymmetric equation of motion for a gaugino has a cubic gaugino coupling, which vanishes only in the Abelian case of a single Dp-brane. The perfect square structure underlying these features of the Dp-branes is a consequence of the superspace geometry. These results are quite general; they apply to type-IIB theory and to the KKLT scenario. They support and further develop the proposal made in Ref. [18].

\section{GAUGINOS IN DP-BRANE ACTIONS}

The Abelian Dp-brane action upon gauge fixing a local $\kappa$ symmetry has an unbroken supersymmetry. Consider the coupling of the background 3-form with the spinor field on the brane in the form given in Ref. [8], in which the gaugefixed action for the Dp-brane is given in approximation quadratic in fermions,

$$
\int d^{p+1} x G_{\mu \nu \rho} \bar{\lambda} \Gamma^{\mu \nu \rho} \lambda
$$

Looking at the complete gauge-fixed Dp-brane action [4,5], one finds that there are no four-fermion terms without 
derivatives on the supersymmetric Dp-brane world volume. But there is also no disagreement with the possibility to add to the action these terms in the form $\left(\bar{\lambda} \Gamma^{\mu \nu \rho} \lambda\right)^{2}$ since they vanish according to Fierz identities, as shown in Refs. [22,23],

$$
\left(\bar{\lambda} \Gamma^{\mu \nu \rho} \lambda\right)^{2}=0 .
$$

The situation changes dramatically when one is trying to figure out the form of the supersymmetric non-Abelian Dpbrane action. The coupling of a background 3-form (1) to gauginos generalizes to

$$
\int d^{p+1} x G_{\mu \nu \rho} \operatorname{Tr} \bar{\lambda} \Gamma^{\mu \nu \rho} \lambda
$$

In the non-Abelian case, the Fierz identity shows that the four-fermion term is not vanishing anymore,

$$
\left(\operatorname{Tr} \bar{\lambda} \Gamma^{\mu \nu \rho} \lambda\right)^{2} \neq 0 .
$$

What is the generalization of the action (1) to the nonAbelian case? The answer is defined by a supersymmetry of the effective low-energy action of supergravity with Dpbrane sources. We will show below that the four-fermion terms with nonderivative coupling shown in Eq. (4) must be present.

When a Dp-brane is described as a local source to the bulk supergravity action, the issue of $\delta^{9-p}(0)$ divergences is important and has to be dealt with, as explained in Ref. [18] in the case of D7-branes with $\delta^{2}(0)$ divergences. Following Refs. [19-21], the authors of [18] argue that the infinite terms cancel due to the presence of four-fermion terms, whereas the finite terms also involve bilinear as well as four-fermion gaugino coupling.

We will start with the space filling coincident D9-branes where the issue of the existence/nonexistence of the fourfermion gaugino term on the Dp-brane world volume is disentangled from the issue of divergences.

\section{FOUR-FERMION INTERACTION IN 4D EINSTEIN-YANG-MILLS SUPERGRAVITY}

In general 4D supergravity, the role of gaugino condensates with regard to spontaneous breaking of local supersymmetry was explained in Ref. [24] based on the properties of the auxiliary fields $F^{\alpha}$ of the chiral multiplets discovered in Ref. [25]. The supergravity action is quadratic in auxiliary fields

$$
\mathcal{L}_{\text {aux }}=-F^{\alpha} g_{\alpha \bar{\beta}} \bar{F}^{\bar{\beta}} \equiv|F|^{2} .
$$

Here, the on-shell value of auxiliary field is

$$
F^{\alpha}=-e^{K / 2} g^{\alpha \beta} \bar{\nabla}_{\bar{\beta}} \bar{W}+\frac{1}{4} \bar{f}_{A B \bar{\beta}} g^{\bar{\beta} \alpha} \bar{\lambda}^{A} P_{L} \lambda^{B},
$$

where we use Eq. (3.14) and in Eq. (4.4) in Ref. [25], but we employ here notation of Ref. [26]. As in Eq. (3) in Ref. [24], we omit the fermions from the chiral multiplets here and only keep fermions from the Yang-Mills multiplets.

Equations (5) and (6) give the first indication of the perfect square nature of the two-fermion and four-fermion coupling in the action. This phenomenon is very general ${ }^{1}$ and underlines the related examples in 10D theory and in supersymmetry breaking by Dp-branes, which we will discuss below.

\section{FOUR-FERMION INTERACTION IN 10D EINSTEIN-YANG-MILLS SUPERGRAVITY}

Once the Dp-brane is added as a local source to the 10D supergravity action, half of supersymmetry is broken. An effective supergravity action describing this situation in an example of a single D9-brane is the action of the 10D Einstein-Maxwell supergravity [23]. The gravitational multiplet includes the graviton, gravitino, dilatino, a 2-form field, and a dilaton: $e_{\mu}^{m}, \psi_{\mu}, \chi, A_{\mu \nu}, \phi$. A matter Maxwell multiplet includes an Abelian vector and a gaugino: $A_{\mu}, \lambda$.

The total Einstein-Maxwell supergravity action with $\mathcal{N}=110 \mathrm{D}$ supersymmetry is [23]

$$
\begin{aligned}
e^{-1} \mathcal{L}^{\mathrm{EM}}= & -\frac{1}{2} R-\frac{3}{4} \phi^{-\frac{3}{2}}\left(F_{\mu \nu \rho}^{M}-\frac{\sqrt{2}}{24} \phi^{\frac{3}{4}} \bar{\lambda} \Gamma_{\mu \nu \rho} \lambda\right)^{2} \\
& -\frac{9}{16}\left(\frac{\partial_{\mu} \phi}{\phi}\right)^{2}-\frac{1}{4} F_{\mu \nu}^{2}-\frac{1}{2} \bar{\lambda} \gamma^{\mu} D_{\mu} \lambda+\cdots
\end{aligned}
$$

Here, terms with ... depend on the gravitino $\psi_{\mu}$ and on a dilatino $\chi$. The 3-form field is $F_{\mu \nu \rho}^{M}=\partial_{[\mu} A_{\nu \rho]}-\frac{1}{\sqrt{2}} A_{[\mu} F_{\nu \rho]}$, and $F_{\mu \nu}=\partial_{\mu} A_{\nu}-\partial_{\nu} A_{\mu}$. The four-fermion term here is vanishing in the Abelian case of just one matter multiplet (2). Therefore, the perfect square term in (7) is optional; one could have had it in the form without a four-fermion coupling, in which case it would not look like a perfect square term. This is exactly the case in the EinsteinMaxwell supergravity [23], for which, instead of the first line of the action (7), one has

$$
-\frac{1}{2} R-\frac{3}{4} \phi^{-\frac{3}{2}}\left(\left(F_{\mu \nu \rho}^{M}\right)^{2}-\frac{\sqrt{2}}{12} \phi^{\frac{3}{4}} F^{\mu \nu \rho M} \bar{\lambda} \Gamma_{\mu \nu \rho} \lambda\right)
$$

and the four-fermion gaugino term is absent.

The D9-brane action at the level at which we neglect higher-derivative nonlinear terms of the Born-Infeld nature is precisely the supersymmetric Maxwell action in the background of $\mathcal{N}=1$ supergravity. Therefore, the supersymmetric action (7) can be viewed as an effective

\footnotetext{
${ }^{1}$ I am grateful to S. Ferrara for explaining this to me.
} 
low-energy approximation of the single D9-brane interacting with gravity, which has $\mathcal{N}=110 \mathrm{D}$ supersymmetry.

If, on the contrary, we would like to have a whole nonlinear Dp-brane action in the background of type-IIB supergravity, we would be able to start with a $\kappa$-symmetric action [1-3], gauge fix local fermionic $\kappa$ symmetry [4,5], and identify the fermionic terms on the Dp-brane. For a single Dp-brane, the four-fermion terms are absent, in agreement with (2). Meanwhile, a complete $\kappa$-symmetric action for coinciding multiple Dp-branes, which are supposed to describe a nonperturbative gaugino condensation, is not known.

Therefore, we can look at the gaugino condensation problem from the perspective of the Einstein-Yang-Mills 10D supergravity [27] but correct the omission of the fourgaugino term there (we skip terms depending on the gravitino and dilatino):

$$
\begin{aligned}
e^{-1} \mathcal{L}^{\mathrm{EYM}}= & -\frac{1}{2} R-\frac{3}{4} \phi^{-\frac{3}{2}}\left(F_{\mu \nu \rho}^{\mathrm{YM}}-\frac{\sqrt{2}}{24} \phi^{\frac{3}{4}} \operatorname{Tr} \bar{\lambda} \Gamma_{\mu \nu \rho} \lambda\right)^{2} \\
& -\frac{9}{16}\left(\frac{\partial_{\mu} \phi}{\phi}\right)^{2}-\frac{1}{4} \operatorname{Tr}\left(F_{\mu \nu}^{\mathrm{YM}}\right)^{2}-\frac{1}{2} \operatorname{Tr} \bar{\lambda} \gamma^{\mu} D_{\mu} \lambda .
\end{aligned}
$$

The 3-form field is now $F_{\mu \nu \rho}^{\mathrm{YM}}=\partial_{[\mu} A_{\nu \rho]}-\frac{1}{\sqrt{2}} \operatorname{Tr}\left(A_{[\mu} F_{\nu \rho]}-\right.$ $\left.\frac{2}{3} A_{[\mu} A_{\nu} A_{\rho]}\right)$ and $F_{\mu \nu}^{\mathrm{YM}}=\partial_{\mu} A_{\nu}-\partial_{\nu} A_{\mu}+\left[A_{\mu}, A_{\nu}\right]$. The fourfermion term here is not vanishing in the non-Abelian case; see (4).

In Ref. [27], the four-fermion gaugino term was omitted even in the non-Abelian case, which was corrected in Ref. [28]. The emphasis in Ref. [28] was on the fact that the corresponding four-fermion term originates from a heterotic string theory in which the computation of the lowenergy scattering of gauginos has revealed its presence.

\section{GEOMETRIC SUPERSPACE ORIGIN OF THE PERFECT SQUARE}

One could perform a brute force computation of the local supersymmetry of the Chapline-Mantone action [27] (or one can compute the four-fermion amplitude in the heterotic string) and find out that the four-fermion gaugino term is required. For example, one can show that the terms with two gauginos and a gravitino and a dilatino, $\lambda^{2} \psi \chi$, and the terms with two gauginos and two dilatinos, $\lambda^{2} \chi^{2}$, in the non-Abelian case have a variation of the form

$$
\delta \mathcal{L}^{M} \sim \operatorname{Tr} \bar{\lambda} \gamma^{a b c} \lambda \bar{\chi} X^{a b c a^{\prime} b^{\prime} c^{\prime}} \epsilon \operatorname{Tr} \bar{\lambda} \gamma^{a^{\prime} b^{\prime} c^{\prime}} \lambda,
$$

where $X^{a b c a^{\prime} b^{\prime} c^{\prime}}$ is a complicated combination of the $\gamma$ matrices. In Abelian case, a cubic in the $\lambda$ expression $\bar{\lambda} \gamma^{a b c} \lambda \gamma_{a b} \lambda$ vanishes, and it can be used to show that $\delta \mathcal{L}^{M}=0$ in the $\lambda^{4} \chi \epsilon$ sector. However, in the non-Abelian case, the expression in (10) does not vanish, and the cooperation with the variation from the four-fermion term $\left(\operatorname{Tr} \bar{\lambda} \gamma^{a b c} \lambda\right)^{2}$, with a specific factor in front, is required.
This can be confirmed by a laborious work, and one would also notice the following. Once the $(\operatorname{Tr} \lambda \lambda)^{2}$ term with the correct coefficient is added to the action, the 3 -form and bilinear gaugino form a perfect square as shown in Eq. (9). In this procedure, the appearance of the perfect square in the action is not clear; it looks like an accident.

However, there is a geometric reason for the perfect square in Einstein-Yang-Mills 10D supergravity in the context of the superspace geometry: the perfect square in the action is a square of the superspace torsion tensor at $\theta=0$, where $\theta$ is the Grassmann coordinate of the superspace.

The 10D superspace was constructed in Refs. [29-31]. We represent it here in slightly more convenient notation. One starts with a superspace with coordinates $z^{M}=$ $\left(x^{\mu}, \theta^{m}\right)$. In this superspace, we introduce the vielbein $E^{A}$, the spin connection $\omega_{A}{ }^{B}$, the 2-form potential $B$, and the Yang-Mills 1-form $A$. The tangent space indices $A$ and $B$ split into bosonic indices $a$ and $b$ and fermionic indices $\alpha$ and $\beta$. We define an exterior derivative covariant with respect to general superspace coordinate transformations as well as local Lorentz and local Yang-Mills transformations: $D=d+\omega+A$. The standard definition of the torsion and curvature in the superspace follows, as do a 3-form and a Yang-Mills 2-form; see Eqs. (3)-(6) in Refs. [29] for details. The torsion $T^{A}$, the curvature $R_{A}{ }^{B}$, the supergravity 3 -form $H$, and the Yang-Mills 2-form $F$ satisfy the superspace Bianchi identities (BIs)

$$
\begin{aligned}
D T^{A} & =-E^{B} \wedge{R_{B}{ }^{A},}_{D R_{A}{ }^{B}=0,} \\
D H & =\operatorname{Tr} F \wedge F, \quad D F=0 .
\end{aligned}
$$

For example, the torsion 2-form defines the torsion tensor

$$
T^{A}=\frac{1}{2} E^{B} \wedge E^{C} T_{C B}^{A} .
$$

To find the solutions of the BI, one has to impose certain constraints on the coupled Einstein-Yang-Mills superspace, for example, that $T_{\alpha \beta}^{a}=-i \gamma_{\alpha \beta}^{a}$, etc., as shown in Table 1 in Ref. [29]. The solution of BI was presented in Refs. [30,31] and is given in terms of the following superfields. There is a superfield with the first component, which is a dilaton, $\varphi(x)$, and all higher components are computed by the repeated action of spinorial derivatives $D_{\alpha}$. There is a YangMills (YM) superfield with the first component being a gaugino field $\lambda(x)$. Finally, one more superfield is required with the first component being a combination of the 3 -form and a gaugino bilinear,

$$
Y_{a b c} \equiv-\frac{1}{72} H_{a b c}-\frac{2}{3} \Lambda_{a b c},
$$

where 


$$
\operatorname{Tr} \lambda_{\alpha} \lambda_{\beta} \equiv \gamma_{\alpha \beta}^{a b c} \Lambda_{a b c}
$$

The component of the superspace torsion $T_{a \beta}{ }^{\gamma}(x, \theta)$ which solves the superspace BI depends both on the dilaton superfield $\varphi(x, \theta)$ and on the superfield $Y_{a b c}(x, \theta)$,

$$
T_{a \beta}^{\gamma}(x, \theta)=-\frac{1}{2}\left(\gamma^{b} \gamma_{a}\right)_{\beta}^{\gamma} D_{b} \varphi-\Gamma_{a}^{b c d} Y_{b c d}
$$

There is also another third-rank antisymmetric tensor superfield, $K_{a b c}=-\frac{1}{72} H_{a b c}-\frac{i}{3} \Lambda_{a b c}-\frac{5 i}{2} \chi_{a b c}$, which includes also a dilatino bilinear $\chi_{a b c}$ and has a mix of $H_{a b c}$ with $\Lambda_{a b c}$, which is different from the one in $Y_{a b c}$. It is a second spinorial derivative of the dilaton superfield, and it does not have superspace tensor properties like $Y_{a b c}$, which represents the superspace torsion.

The component action perfect square term in (9) originates from the square of the superspace torsion term

$$
\left.\mathcal{L}^{\mathrm{EYM}} \supset T_{a \beta}^{\gamma} T_{\gamma}^{a \beta}(x, \theta)\right|_{\theta=0} \sim Y_{a b c} Y^{a b c}(x),
$$

where we have neglected the terms with derivatives on a dilaton which are present in a superspace torsion (15).

The Einstein equation for the 10D curvature also depends only on the same perfect square

$$
R_{10} \supset Y_{a b c} Y^{a b c}(x)+\cdots
$$

Thus, the superspace geometry clearly explains that supersymmetry does require the four-fermion term $\left(\operatorname{Tr} \bar{\lambda} \Gamma^{\mu \nu \rho} \lambda\right)^{2}$ in the action, and moreover, it has to come with the coefficient which forms a perfect square in the action, as shown in Eqs. (9) and (16). Therefore, the solution with gaugino condensation, which makes the first component of the superfield vanish,

$$
Y_{a b c}=-\frac{1}{72} H_{a b c}-\frac{2}{3}\left\langle\Lambda_{a b c}\right\rangle=0,
$$

is consistent with local supersymmetry of the theory. In such a case, the term in the action $Y_{a b c} Y^{a b c}$ does not contribute to the vacuum energy.

Thus, in the case of a noncompact geometry, described by the superspace in Refs. [29-31], gaugino condensation requires a nonvanishing 3-form. It backreacts so that the total square vanishes. This is in agreement with the earlier observations on this in Ref. [28].

We conclude that if a complete supersymmetric nonlinear non-Abelian Dp-brane action is available it must have on its world volume the four-fermion term to provide the perfect square term shown in Eq. (9) in the D9 case. This is necessary to satisfy a condition of supersymmetry of a non-Abelian vector supermultiplet interacting with supergravity.

\section{GAUGINO EQUATION OF MOTION ON DP-BRANE}

For the D7-brane, it is more difficult to use the perfect square structure of the action in the component form in (9) or in the superspace form in (16), as we did for the D9-brane. Indeed, the 10D action originates from the supergravity action $\int d^{10} x G_{a b c} G^{a b c}$, whereas the gauginodependent terms originate from the brane action $\int d^{8} x G_{a b c} \operatorname{Tr} \bar{\lambda} \gamma^{a b c} \lambda$ and $\int d^{8} x\left(\operatorname{Tr} \bar{\lambda} \gamma^{a b c} \lambda\right)^{2}$. Therefore, we have to deal with some singular terms from the local sources.

Of course, one can rely on the simple fact that the fourfermion term existing on D9 should remain on D7 after dimensional reduction. To support this argument that the term $\int d^{8} x\left(\operatorname{Tr} \bar{\lambda} \gamma^{a b c} \lambda\right)^{2}$ must be present in D7-brane action, we may look at the supersymmetric equation of motion for the gaugino which interacts with the supergravity background. Such an equation was derived in the context of the superspace geometry [29-31] in the noncompact 10D space.

The manifestly supersymmetric field equation for the gaugino is presented in Eq. (21) in Ref. [31]. It depends on the background 3-form only via the torsion tensor $Y_{b c d}$,

$\gamma^{a} \hat{D}_{a} \lambda=3 Y_{a b c} \gamma^{a b c} \lambda=3\left(-\frac{1}{72} H_{a b c}-\frac{2}{3} \Lambda_{a b c}\right) \gamma^{a b c} \lambda$.

Here, $\hat{D}_{a}=D_{a}-3 D_{a} \phi$. The three-gaugino coupling term in the manifestly supersymmetric field equation for a gaugino in (19) is given by

$$
\gamma^{a b c} \Lambda_{a b c} \lambda \sim \operatorname{Tr}\left(\bar{\lambda} \gamma_{a b c} \lambda\right) \gamma^{a b c} \lambda,
$$

since $\Lambda_{a b c} \sim \operatorname{Tr} \bar{\lambda} \gamma_{a b c} \lambda$. This cubic gaugino coupling vanishes in the Abelian case in which $\left(\bar{\lambda} \gamma_{a b c} \lambda\right) \gamma^{a b} \lambda=0$, but in the non-Abelian case, the cubic coupling is not vanishing. This confirms unambiguously the presence of the fourgaugino coupling on a non-Abelian Dp-brane.

\section{STRING THEORY AND FINITE VOLUME ISSUES}

The next conceptual step away from supergravity, defined in the infinite 10D space-time, was an observation in Refs. [19-21] about an M theory compactified on a onedimensional finite-size interval. The localized source in this case of the form $\sim \delta\left(x^{11}\right)$ is present; however, it is argued in Refs. [19-21] that supersymmetry requires the presence of a quartic gaugino term in the action. In Ref. [21], it was suggested to shift the field strength 4-form $G_{I J K 11} \equiv G_{a b c}$ by a term supported at the boundary and bilinear in the gauginos and to define the modified 3 -form field as follows:

$$
\tilde{G}_{a b c}=G_{a b c}+c \delta\left(x_{11}\right) \Lambda_{a b c} .
$$


The effective action depends on the 3-form $G_{a b c}$ only via the perfect square,

$$
\mathcal{L} \supset\left(\tilde{G}_{a b c}\right)^{2} .
$$

The presence of this four-fermion term in the action precisely cancels the divergence due to a perfect square structure analogous to the one we explained above. In a sense, the 10D non-Abelian supersymmetry is an underlying reason for the four-fermion term in the action.

The four-fermion term plays an important role in gaugino condensation, as shown in Ref. [18]. The authors have proposed that gaugino condensation in D7-branes should be described by the effective action in their Eqs. (39)-(41). The presence of the four-gaugino coupling on the D7-brane, according to Ref. [18], resolves the issues of divergences and allows a comparison with the 4D theory.

In view of the compact volume of compactification, things are more complicated than in the case of the infinite six-dimensional (6D) space described above in our Eqs. (9) and (16) for 10D Einstein-Yang-Mills supergravity and for non-Abelian D9-branes.

However, the perfect square structure of the effective action in non-Abelian D7 is not just reminiscent of heterotic strings or M theory compactified on the interval; the perfect square terms in the action in all of these cases are actually inherited from the 10D Einstein-Yang-Mills superspace geometry.

If the D9-branes are compactified on a torus of a square area $A_{T}^{2}=L^{2}$, and the total internal space is $X=\Sigma \times T^{2}$ with $V_{\Sigma}$ being the finite volume of the $\Sigma$ manifold, one would expect that our action (9) would lead to Eqs. (39)(41) in Ref. [18]. This would result in cancellation of the relevant infinities and in clarification of the gaugino condensation on D7-branes.

Specifically, once the conjectured supersymmetric action of D7 non-Abelian branes is derived from a compactification of D9-branes on $T^{2}$, it is plausible that the remaining finite terms in the action are of the form given in Eq. (41) in Ref. [18],

$$
-g_{s}\left|G_{3}^{(0)}+\frac{\lambda \lambda}{\sqrt{g_{s}} A_{T^{2}}} \bar{\Omega}_{3}\right|^{2} .
$$

If one admits the terms bilinear in spinors on a non-Abelian D7-brane, also the quartic terms must be present, as follows from (9) and from (19) and (20).

The technical issues with singularities may still require some clarification. The issue of supersymmetry in singular spaces is complicated, as was shown in the past in a different context in Ref. [32].

\section{RELATION TO 4D SUPERSYMMETRY}

It is known that type-IIB supergravity with local sources, which involve calibrated Dp-branes and O planes, leads to a
4D $\mathcal{N}=1$ supergravity. String theory constructions in 10D space compactified on calibrated manifolds were mostly compared with $4 \mathrm{D} \mathcal{N}=1$ supergravity with chiral multiplets. The dictionary between the Kahler potential $K(z, \bar{z})$ and the holomorphic superpotential $W(z)$ and 10D theory was established. Moreover, in the presence of pseudocalibrated anti-Dp branes, the 4D supergravity also involves a nilpotent chiral superfield; see Ref. [33] and references therein. Meanwhile, the presence of the vector multiplets in 10D supergravity, which live on the Dp-branes, can also be studied. In particular, one may associate the known structure of $4 \mathrm{D}$ theory with $\mathcal{N}=1$ vector multiplets with 10D theory with Dp-branes. The dictionary between string theory models and $\mathcal{N}=14 \mathrm{D}$ supergravity in such a case will also involve the holomorphic vector coupling functions $f_{A B}(z)$.

In Ref. [18], evidence was given that the authors' proposal for the D7-brane action with four-fermion terms with careful account of a compact volume of compactification leads to a 4D action with the vector multiplets.

The action of $\mathcal{N}=1$ vector multiplets interacting with supergravity has the following relevant terms [26]:

$$
\begin{aligned}
& -\frac{1}{4} \operatorname{Re} f(z)\left(F_{\mu \nu}^{A} F^{\mu \nu A}-2 \bar{\lambda} \gamma^{\mu} D_{\mu} \lambda^{A}\right)+\cdots \\
& +\frac{1}{4} e^{\frac{K(z, \bar{z})}{2}} f_{, \alpha} \bar{\nabla}{ }^{\alpha} \bar{W} \bar{\lambda}^{A} P_{L} \lambda^{A}+\text { H.c. } \\
& +\frac{3}{64}\left[\operatorname{Re} f(z)\left(\bar{\lambda}^{A} \gamma^{\mu} \gamma_{*} \lambda^{A}\right]^{2}\right. \\
& -\frac{1}{16} f_{, \alpha} \bar{f}, \bar{\lambda}^{A} P_{L} \lambda^{A} \bar{\lambda}^{B} P_{R} \lambda^{B},
\end{aligned}
$$

where we consider the case with the diagonal holomorphic vector coupling $f_{A B}(z)=f(z) \delta_{A B}$. This expression is in agreement with Eqs. (5) and (6) above. Clearly, 4D supergravity if derived from 10D string theory with branes, should have no singularities, and must have four-fermion gaugino coupling.

If $f(z)$ is a gauge coupling on $\mathrm{N}$ coincident $\mathrm{D} 7$-branes and 10D theory is compactified on a compact $T^{2} \times T^{2} \times T^{2}$ space with finite volume, one finds that, indeed, the $4 \mathrm{D}$ action (24) with terms bilinear and quartic in gaugino follows from Eq. (23), as observed in Ref. [18].

In conclusion, here, we have shown that the perfect square term in the 10D Einstein-Yang-Mills supergravity originates from the superspace geometry. Namely, the action includes a square of the first component of the supertorsion superfield, as shown in Eq. (16), which is a unique combination of the 3 -form and a gaugino bilinear in Eq. (13). The same torsion superfield is present in the gaugino equation of motion consistent with supersymmetry, as shown in (19). This means that a cubic gaugino coupling is present in the gaugino equation of motion, and the quartic fermion coupling must be present on a 
non-Abelian Dp-brane, as follows from the superspace geometry.

A better understanding of the impact of the four-fermion terms in condensing D7-branes, particularly with regard to a finite volume of compactification, would be useful for the purpose of relating 10D string theory to cosmological observations described by the 4D physics.

\section{ACKNOWLEDGMENTS}

I would like to thank S. Ferrara, M. Dine, A. Linde, E. McDonough, B. Nillsson, and A. Tseytlin for helpful comments and discussions. This work is supported by SITP, by the NSF Grant No. PHY-1720397, and by the Simons Foundation Origins of the Universe program (Modern Inflationary Cosmology Collaboration).
[1] M. Cederwall, A. von Gussich, B. E. W. Nilsson, and A. Westerberg, The Dirichlet super three-brane in tendimensional type IIB supergravity, Nucl. Phys. B490, 163 (1997).

[2] M. Cederwall, A. von Gussich, B. E. W. Nilsson, P. Sundell, and A. Westerberg, The Dirichlet super p-branes in tendimensional type IIA and IIB supergravity, Nucl. Phys. B490, 179 (1997).

[3] E. Bergshoeff and P. K. Townsend, Super D-branes, Nucl. Phys. B490, 145 (1997).

[4] M. Aganagic, C. Popescu, and J.H. Schwarz, Gauge invariant and gauge fixed D-brane actions, Nucl. Phys. B495, 99 (1997).

[5] E. Bergshoeff, R. Kallosh, T. Ortin, and G. Papadopoulos, Kappa symmetry, supersymmetry and intersecting branes, Nucl. Phys. B502, 149 (1997).

[6] D. Marolf, L. Martucci, and P. J. Silva, Fermions, T duality and effective actions for D-branes in bosonic backgrounds, J. High Energy Phys. 04 (2003) 051.

[7] P. G. Camara, L. E. Ibanez, and A. M. Uranga, Flux-induced SUSY-breaking soft terms on D7-D3 brane systems, Nucl. Phys. B708, 268 (2005).

[8] L. Martucci, J. Rosseel, D. Van den Bleeken, and A. Van Proeyen, Dirac actions for D-branes on backgrounds with fluxes, Classical Quantum Gravity 22, 2745 (2005).

[9] D. Baumann, A. Dymarsky, S. Kachru, I. R. Klebanov, and L. McAllister, D3-brane potentials from Fluxes in AdS/CFT, J. High Energy Phys. 06 (2010) 072.

[10] A. Dymarsky and L. Martucci, D-brane non-perturbative effects and geometric deformations, J. High Energy Phys. 04 (2011) 061.

[11] J. Moritz, A. Retolaza, and A. Westphal, Toward de Sitter space from ten dimensions, Phys. Rev. D 97, 046010 (2018).

[12] F. F. Gautason, V. Van Hemelryck, and T. Van Riet, The tension between 10D supergravity and dS uplifts, Fortschr. Phys. 67, 1800091 (2019).

[13] M. Cicoli, S. de Alwis, A. Maharana, F. Muia, and F. Quevedo, De Sitter vs quintessence in string theory, Fortschr. Phys. 67, 1800079 (2019).

[14] Y. Akrami, R. Kallosh, A. Linde, and V. Vardanyan, The landscape, the swampland and the era of precision cosmology, Fortschr. Phys. 67, 1800075 (2019).
[15] R. Kallosh, A. Linde, E. McDonough, and M. Scalisi, de Sitter Vacua with a Nilpotent Superfield, Fortschr. Phys. 67, 1800068 (2019).

[16] R. Kallosh, A. Linde, E. McDonough, and M. Scalisi, 4d models of dS uplift in KKLT, Phys. Rev. D 99, 046006 (2019).

[17] R. Kallosh, A. Linde, E. McDonough, and M. Scalisi, dS Vacua and the Swampland, arXiv:1901.02022.

[18] Y. Hamada, A. Hebecker, G. Shiu, and P. Soler, Brane gaugino condensate in 10d, arXiv:1812.06097.

[19] P. Horava and E. Witten, Heterotic and type I string dynamics from eleven-dimensions, Nucl. Phys. B460, 506 (1996).

[20] P. Horava and E. Witten, Eleven-dimensional supergravity on a manifold with boundary, Nucl. Phys. B475, 94 (1996).

[21] P. Horava, Gluino condensation in strongly coupled heterotic string theory, Phys. Rev. D 54, 7561 (1996).

[22] B. E. W. Nilsson, Simple ten-dimensional supergravity in superspace, Nucl. Phys. B188, 176 (1981).

[23] E. Bergshoeff, M. de Roo, B. de Wit, and P. van Nieuwenhuizen, Ten-dimensional Maxwell-Einstein supergravity, its currents, and the issue of its auxiliary fields, Nucl. Phys. B195, 97 (1982).

[24] S. Ferrara, L. Girardello, and H. P. Nilles, Breakdown of local supersymmetry through gauge fermion condensates, Phys. Lett. 125B, 457 (1983).

[25] E. Cremmer, S. Ferrara, L. Girardello, and A. Van Proeyen, Yang-Mills theories with local supersymmetry: Lagrangian, transformation laws and SuperHiggs effect, Nucl. Phys. B212, 413 (1983).

[26] D.Z. Freedman and A. Van Proeyen, Supergravity (Cambridge University Press, Cambridge, England, 2012).

[27] G. F. Chapline and N. S. Manton, Unification of Yang-Mills theory and supergravity in ten-dimensions, Phys. Lett. 120B, 105 (1983).

[28] M. Dine, R. Rohm, N. Seiberg, and E. Witten, Gluino condensation in superstring models, Phys. Lett. 156B, 55 (1985).

[29] R. E. Kallosh and B. E. W. Nilsson, Scale invariant $d=10$ superspace and the heterotic string, Phys. Lett. 167B, 46 (1986). 
[30] B. E. W. Nilsson and A. K. Tollsten, The geometrical offshell structure of pure $N=1 d=10$ supergravity in superspace, Phys. Lett. 169B, 369 (1986).

[31] B. E. W. Nilsson and A. K. Tollsten, Superspace formulation of the ten-dimensional coupled Einstein Yang-Mills system, Phys. Lett. B 171, 212 (1986).
[32] E. Bergshoeff, R. Kallosh, and A. Van Proeyen, Supersymmetry in singular spaces, J. High Energy Phys. 10 (2000) 033.

[33] R. Kallosh and T. Wrase, dS Supergravity from 10d, Fortschr. Phys. 67, 1800071 (2019).

[34] S. Kachru, R. Kallosh, A. D. Linde, and S. P. Trivedi, de Sitter vacua in string theory, Phys. Rev. D 68, 046005 (2003). 